\title{
About the long-term coordinated variations of the activity, radius, total irradiance of the Sun and the Earth's climate
}

\author{
Habibullo I. Abdussamatov \\ Pulkovo Observatory, Saint Petersburg, Russia
}

The 11-year cycle represents a simultaneous parallel change in both the activity level and the total irradiance of the Sun. So, in case of variations of the amplitude of the activity level - a power of a cycle - the amplitude of solar irradiance variations is expected to change correspondingly. The identical correlated course of the long-term variations of activity and luminosity of the Sun on the secular timescale has been observed earlier by Eddy (1976), and Borisenkov (1988). Moreover, according to the data of Borisenkov (1988), in each of 18 deep Maunder-type minima of solar activity, revealed over the span of the last 7500 years, the cooling of climate had been observed, while warming occurred during the periods of high maxima. Thus, the integral radiation has always been essentially higher at the maximum, and it had noticeably decreased at the minima. Therefore, quasi-periodic variations of the solar activity during both the 11-year cycle and 80 - and 200-year cycles are accompanied by proportional variations of the integral flux of solar radiation, which result in geophysical effects. The main cause of climate change during the last millennia is the corresponding cyclic variation of the $80-$ and 200year component of irradiance correlated with activity. That is why, the contemporary is not anomalous but is ordinary secular global warming (Aguilar 2003; Reid 2000), as well as previous similar cases of warming during the periods of secular activity growth is still mainly connected with an increase of the secular component of solar irradiance variation.

Recent observations Ulrich (1995) and Noel (1997, 2001, 2002), show that the solar radius variations within an 11-year cycle has the correlated identically with the activity level variations, although a contrary result with smaller amplitude was obtained by Laclare (1996). Furthermore, revealed that the larger radius is connected with high activity level, and a smaller one with low activity level (Basu 1998). The results of Sveshnikov (2002) have ultimately confirmed the reality of close connection between the change of activity level during a cycle and the course of radius variations in both phase and amplitude. In particular, during 11-year cycles with an increased activity level, larger amplitude of radius variations is generally observed, while during the cycles with a decreased activity level - the lower amplitude, i.e. the courses of 11-year variations of both the radius and the activity level are mutually correlated and parallel to each other. A presence of 80-year cycle in the radius variations (Sveshnikov 2002, Parkinson 1980, Gilliland 1981) together with secular climate variations is an additional argument in favor of presence of secular component in the solar irradiance variations. In the phase of maxima of 11-, 80and 200-year cycles has correspondingly larger radius and higher luminosity, while in the phase of minima of these cycles an opposite picture is observed.

Observed correlated long-term identical variations of radius, irradiance and activity, which require enormous energy resources for a rather long time, are, in our opinion, the consequence of the same processes, taking place in the innermost solar interior, and are coordinated by a global variation of the whole inner structure of the Sun, caused by changes in the state of energy-releasing core, its temperature, and, probably, its rotation 
rate in the course of cyclic activity of the star (Abdussamatov 2003). Variation of energy release from the core and corresponding temperature change lead to disturbance of the solar equilibrium, determined by the balance of forces of the inner pressure and gravity. An increase of energy release in the core is accompanied by the growth of temperature, which result in unavoidable global heating of the Sun, and corresponding increase of its radius and total irradiance with the effective temperature remaining practically constant. Therefore, cyclic variations of the "solar constant" result from corresponding change of the emitting area, i.e. $W_{\odot}=\left(\Delta R_{\odot} / R_{\odot}\right) /\left(\Delta S_{\odot} / S_{\odot}\right)=0.5$. Hence, the amplitude of the 11-year radius variation should be limited to $\Delta R<0,5$ ".

Thus, possible long-term smooth quasi-periodic oscillations of the core's energy release, accompanied by the corresponding temperature changes, can lead to cyclic global reconstructions of the whole Sun, induced radial pulsations around mean value of the radius, and, therefore, to variations of the "solar constant", proportional to the relative change of the radius squared. Such long-term global variations of the whole Sun, caused by the corresponding changes in the temperature of the core, can catalyze generation of an activity cycle. Increase of the core's temperature and corresponding expansion of the whole Sun can catalyze the raise of activity, while decrease of the core's temperature will catalyze a drop in activity, with the amplitude of the central temperature variations determining the power of a cycle. If amplitude of the core's temperature fluctuations is small, weak cycles with a low-amplitude activity level will be developed, while higher amplitudes result in more powerful cycles. An absence or a rather low amplitude of the temperature fluctuations can lead to a deep minimum of both activity and luminosity of a Maunder-type. Hence, solar activity can acquire additional energy released in the core.

Summarizing, observed cyclicities in solar variations are determined by corresponding quasi-periodic changes in both activity and size (and, therefore, total irradiance). That is why, one can expect that in the nearest future (in accordance with expected decay of the activity and irradiance secular cycle) regular secular decrease of the Earth temperature should replace the contemporary not anomalous but regular secular global warming.

\section{References}

Abdussamatov H.I. 2003, Petersburg fragments of the scientific picture of the Universe, N2. SPbSC RAS. Saint-Petersburg, p.8

Aguilar D. 2003, Harvard-Smithsonian Center for Astrophysics, Press Release, No.: 03-10

Basu D. 1998, Solar Phys., 183, 291

Borisenkov E.P. (ed.) 1988, Climate changes during the last millennium. Leningrad, Gidrometeoizdat

Eddy J.A. 1976, Science, 192, 1189

Gilliland R.L. 1981, Astrophys. J., 248, 1144

Laclare F., Delmas C., Coin J.P., Irban A. 1996, Solar Phys., 166, 211

Noel F. 1997, Astron. Astrophys., 325, 825

Noel F. 2001, Astron. Astrophys., 374, 697

Noel F. 2002, Astron. Astrophys., 396, 667

Parkinson J.H., Morrison L.V., Stephenson F.R. 1980, Nature, 288, 548

Reid G.C. 2000, Space Science Reviews, 94, 1

Sveshnikov M.L., 2002, Sov. Astron. Letters, 28, 133

Ulrich R.K., Bertello L. 1995, Nature, 377, 214 